\title{
Detecting Shape Similarities in 3D Pottery Repositories
}

\author{
Anestis Koutsoudis, George Pavlidis, Christodoulos Chamzas \\ Cultural and Educational Technology Institute/Research Centre 'Athena' \\ Xanthi, Greece \\ akoutsou@ipet.gr,gpavlid@ipet.gr, chamzas@ipet.gr
}

\begin{abstract}
Shape matching is one of the main research procedures performed by archaeologists. Real time 3D computer graphics, 3D digitisation and content based retrieval are technologies that can facilitate the automation of the shape matching process. As pottery plays a significant role in understanding ancient societies, we focus on the development of compact shape descriptors that can be used for content based retrieval of complete or nearly complete $3 D$ pottery replicas. In this work, we present shape descriptors that exploit the axial symmetry feature and attempt to enhance the archaeological study of pottery by using 3D graphics technologies. We evaluated the performance of the descriptors using a $3 D$ pottery ground truth repository as a test bed. We created an experimental 3D pottery search engine and attempted to integrate a content based retrieval mechanism in a 3D virtual reality environment.
\end{abstract}

Keywords-pottery; content based retrieval; shape matching; pottery; MPEG-7; $3 D$ vessel modelling; $3 D$ repositories;

\section{INTRODUCTION}

The application of 3D computer graphics technologies and signal processing in the field of archaeological research is an important step towards the automation of several time consuming procedures. As $3 \mathrm{D}$ digitisation is growing into a common practice in the cultural heritage domain, Web based $3 \mathrm{D}$ model repositories have gained great popularity over the last years [1]-[4]. With the availability of 3D replicas, content based retrieval technologies (CBR) can be put under the services of archaeologists. As CBR is not based on textual metadata, it can detect morphological similarities between artefacts by overcoming, the otherwise expected, multilanguage barriers. Additionally, CBR might offer solutions to archaeological problems related with cataloguing and searching.

In this work, we focus on applying CBR on pottery which is considered as one of the major candidates for 3D digitisation. Artefacts of this domain are considered '3D Scanner Friendly' due to their morphological features (e.g. axial symmetry and low reflectivity surfaces). Additionally, it is a fact that pottery has a remarkable continuity through time and it is used by archaeologists to identify and comprehend various aspects of ancient societies. Based on the previous, we attempted to cerate pottery specific shape descriptors. The descriptors can be extracted from 3D mesh vessel replicas after pre-processing them with a scale and pose normalisation algorithm [5]. We have created a $3 \mathrm{D}$ vessel repository which is composed by both digitised and computer generated models. We have annotated the content of the repository in terms of archaeological features (e.g. shape, type of shape, preservation state) and 3D computer graphics features (e.g. digitised or modelled, texture map availability). We have used this 'ground truth' repository as a testbed for CBR experiments in order to evaluate the performance of the proposed shape descriptors. We have created an experimental Web accessed $3 \mathrm{D}$ pottery CBR search engine. Additionally, we attempted to integrate CBR search engine in an interactive real time 3D environment.

The subsequent sections of this paper are organised as follows: In section 2, we describe the content of our 3D vessel repository. In section 3 , we describe the pottery oriented $3 \mathrm{D}$ shape descriptors. Then, in section 4, we discuss the performance evaluation results. In section 5 , we describe the experimental 3D pottery search engines and we conclude this work in section 6 .

\section{A 3D POTTERY GROUND TRUTH REPOSITORY}

The number of Web accessed 3D repositories is growing dramatically as the demand for 3D models continuously increases. All CBR related research works found in literature use a calibrated $3 \mathrm{D}$ model repository in order to evaluate the performance of their proposed method. These "benchmark" repositories cover 3D models from multiple domains such as airplanes, cars, animals, insects, furniture, plants, mechanical parts (CAD), imaginary beings, etc. On the other hand, the number of publicly available $3 \mathrm{D}$ replicas derived from the cultural heritage is very limited. Thus, the need for creating and calibrating a 3D pottery repository was mandatory. We have gathered a total of 1012 3D pottery models. 94 vessels were digitised using the shape from silhouette and laser triangulation methods, 718 vessels were manually modelled by different research groups and 200 were dynamically generated using the "qp" software tool [6]. The repository covers several shape categories such as ancient Greek (Alabastron, Amphora, Hydria, Kantharos, Lekythos, Psykter, etc), Native American (Jar, Effigy, Bowl, Bottle, etc) and modern pottery.

The dataset was calibrated by annotating each vessel with information related to both archaeological and computer graphics aspects. The archaeological side contains attributes such as the shape and the type of shape, the preservation state, the manufacturing technique that has been used, its attribution, the workshop's name, etc. On the other hand, the computer graphics side provides information about the 3D digitisation 
technique that has been used and the availability of texture map information [5]. The metadata have been organised in a nativeXML database using a custom MPEG-7 compatible schema $[6]$.

\section{3D POTTERY SHAPE DESCRIPTORS}

The shape descriptors proposed in this paper are extracted after applying a scale and pose normalisation algorithm to the 3D mesh [5]. The algorithm takes advantage of the axial symmetry of the vessel and orients it in the 3D space by taking under consideration several morphological features such as the appendages and mouth position.

\section{A. Axial Symmetry Based Shape Descriptor (ASB)}

The data that are used in this descriptor are extracted from the curves that are produced after contouring the vessel's 3D mesh at multiple levels parallel to the $\mathrm{X}-\mathrm{Z}$ plane defined by a right handed 3D Cartesian coordinates system. Those curves are grouped into those that belong the main body of the vessel and those that belong to the appendages. Each curve is described by a number of points and an index list of pointers that organises them into line segments. Circular regression is performed for each curve in both groups. Thus, properties such as the centre of gravity of the best fitted circle, its radius and the circle fitting error are computed. The descriptor is divided into two parts. The first part is used to describe the main body of the vessel while the second encodes the appendages positions in the unit circle.

More specifically, the first part is a $1 \mathrm{D}$ vector which carries the radiuses of the best fitted circle of the curves that belong to the outer shell of the vessel's main body at each contouring level. A graph depiction of this vector is visually similar to a quantized version of the vessel's body outer profile. The second part of the descriptor encodes the properties of all curves that belong to the appendages group in a $2 \mathrm{D}$ binary array. This time, the position of the best fitted circle of each curve is quantized into a predefined area-sector around the unit circle (Fig. 1). The columns of the binary array indicate the sectors while the rows indicate the contouring level in which each curve belongs [5]. In our tests we have selected $\pi / 8$ as the angle step between two consecutive unit circle sectors.

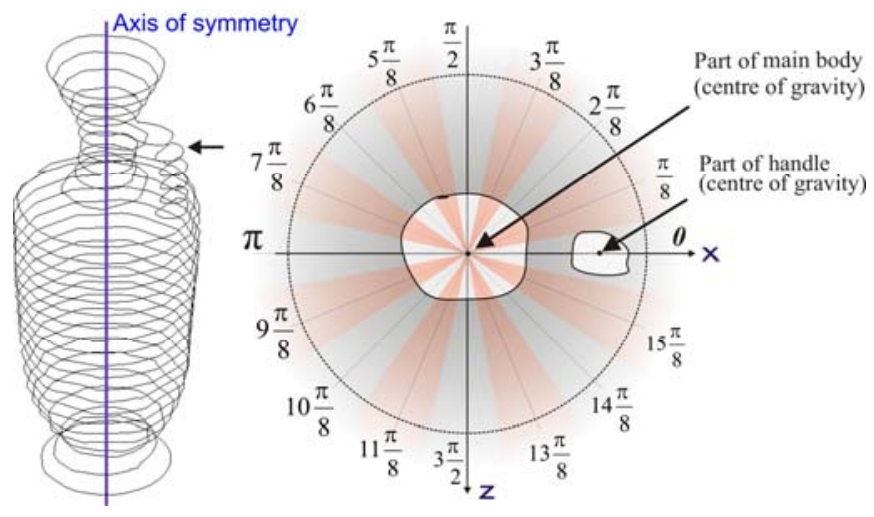

Figure 1. 3D mesh contouring and appendage position-sector matching
The proposed descriptor can be used for partial matching between the main body and the appendages of the vessel. The similarity metric that is used for this descriptor is the normalised sum of the L2 norm for the first part and the hamming distance of the $2 \mathrm{D}$ binary array for the second part [5].

\section{B. Depth Map Image Based Descriptor (DBD)}

The following descriptor is based on the assumption that the main morphological features of a vessel can be captured using a pair of depth map images. The depth map images extraction is performed after the completion of the previously mentioned 3D vessel preprocessing algorithm. A set of two orthographic projection virtual cameras are positioned on the positive sides of the $\mathrm{X}$ and $\mathrm{Z}$ axes of a 3D Cartesian coordinates system. Fig 2. illustrates this virtual camera's set up. The viewpoint direction of both cameras is set towards the vessel's axis of symmetry while the projection planes have an area equal to the side of a unit cube. The surfaces that are not captured on these projection planes are considered supplementary to those being captured. This is due to the rotational symmetry of the vessels and the tubular form of the appendages.

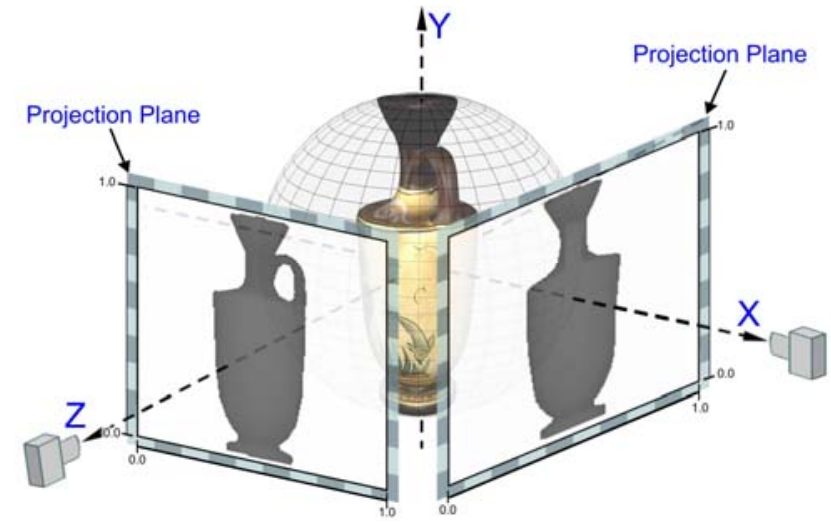

Figure 2. Depth map image capturing virtual set up

For the encoding of the two depth maps, we propose a variant of the Chaouch et al. [7] descriptor. The original descriptor was proposing the use of twelve images captured from the vertices of a dodecahedron. We attempt to exploit the axial symmetry and reduce the size of the descriptor by using only two depth map images. The vessel's surface relief is encoded using characters that represent the surface curvature direction (Fig. 3). Different characters are used to discriminate between the vessel's surface region and the background region.

Thus, a pixel that belongs to the outer-background region is represented by the $b$ character while the $i$ character represents a part of the inner-background region (e.g. background areas surrounded by a handle). Additionally, the vessel's surface relief is encoded using characters that represent the surface curvature direction (Figure 3 ). In our implementation we have used the characters $u, d$ and $s$ which represent a positive line slope (up), a negative line slope (down) and a zero line slope (steady). The surface slope is computed between sequential pixels using their greyscale values. 


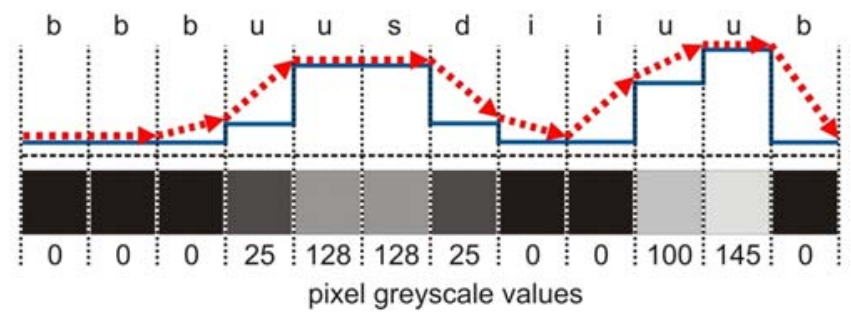

Figure 3. Pixel based character encoding

The two images are encoded into character sequences following a "row by row" and a "column by column" approach. The character strings that are produced by each pixel parsing approach are added together assembling two final cumulative character strings. The Needlman-Wunsch global sequence alignment algorithm is used as the similarity metric between the character sequences [8]. The similarity scores of each final cumulative character string are summed up resulting the total similarity between two vessels.

\section{Shape DESCRIPTORS PERFormanCE EVAluAtion}

In order to evaluate the performance of the proposed descriptors, we performed multiple queries-by-example on our calibrated 3D vessel repository. We have selected five different ancient Greek shapes due to their relatively high population within the database. These were Alabastron (55 objects), Amphora (96 objects), Hydria (26 objects), Lekythos (16 objects) and Psykter (48 objects). Apart from the two proposed descriptors, we evaluated the performance of a generic LightField descriptor proposed by Chen et al. [10]. The descriptor is based on the idea that when two objects look similar from multiple viewpoints then they have to be similar. Thus, Chen et al. [10] propose capturing the object from a hundred different viewpoints using binary images and encoding these images using 2D Zernike moments and 2D Fourier transform. In order to compute the LightField descriptor we have used the software provided by the authors. Additionally, it should also be mentioned that for the "Axial Symmetry Based Shape Descriptor" (ASB) a total of 32 contouring levels has been used while for the "Depth Map Image Based Descriptor" (DBD) we have used depth map images of $32 \times 32$ pixels.

For each one of the chosen shape categories we have calculated the average precision-recall graph by using each vessel as a query object. Then, we computed the overall average precision-recall graph for all shape categories (Fig. 4). Furthermore, for a more complete evaluation, we calculated five performance scalars proposed by the annual 3D Shape Retrieval Contest [9]. Each scalar focuses on different aspects of the descriptor's performance and contributes to a more objective evaluation (Table I). For all scalars, apart the Emeasure, a higher value indicates higher performance.

According to the results, the average discriminating ability of the ASB descriptor is higher than the other two descriptors. This is due to the fact that it exploits the axial symmetry of vessels. The DBD and the LightField descriptor performed almost identically indicating our initial assumption that the main features of a normalised in terms of scale and orientation vessel can be captured by using only two viewpoints. For all three descriptors the NN scalar remains close to 0.9 which indicates that there is a $90 \%$ probability that the first ranked vessel will be of the same shape class like the query-object.

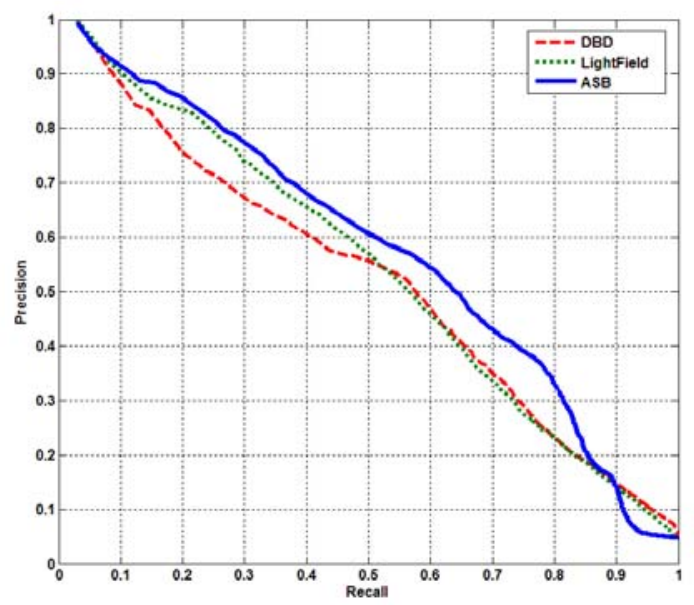

Figure 4. Overall precision-recall graph for all three descriptors

TABLE I. PERFORMANCE SCALARS

\begin{tabular}{|c|c|c|c|c|c|}
\hline $\begin{array}{c}\text { Descriptor } \\
\text { Name }\end{array}$ & $\mathbf{1}^{\text {st }}$ Tier & $2^{\text {nd }}$ Tier & $\begin{array}{c}\text { Averaged } \\
\text { Mean } \\
\text { Precision }\end{array}$ & E-Measure $^{64}$ & NN \\
\hline ASB & 63.20 & 37.60 & 0.57 & 0.48 & 0.9 \\
\hline DBD & 53.55 & 33 & 0.49 & 0.52 & 0.9 \\
\hline LightField & 53.56 & 33.31 & 0.49 & 0.52 & 0.92 \\
\hline
\end{tabular}

\section{EXPERIMENTAL 3D POTTERY SEARCH ENGINES}

We have developed two experimental 3D pottery search engines. The first one is presented through a Website and it is based on 'traditional' graphical user interface components. Hence, the $3 \mathrm{D}$ vessels are depicted using thumbnail images. The user can browse the collection of vessels and have access to the metadata that accompany each vessel. This search engine allows the user to perform queries by uploading a 3D vessel, by sketching a vessel profile using a digital canvas or by using an existing vessel as the query-by-example. Additionally, the search engine allows the performance of queries based on the vessel's metadata. Thus, the user can query the engine to display all vessels that have been digitised or those that carry texture maps. The search engine was built using open source software (PHP, Apache, Java Applets) while the metadata follow a MPEG-7 compatible scheme and are stored in a native-XML database (eXist). The search engine can be found at http://www.ipet.gr/3DPSE.

The second search engine is an initial attempt to integrate the functionality of a CBR system in an interactive real time $3 \mathrm{D}$ virtual reality environment. The proposed integration 
enhances the visitor of the 3D scene with the ability to perform questions such as 'Where else in this scene I can find objects similar to this?'.

By exploiting the fact that complex 3D scenes are composed by hierarchical structures of 3D models, 3D shape descriptors can be computed for each 3D model in the scene and organised in a dedicated database in order to perform CBR.

In this search engine, the user can perform a virtual tour in a 3D reconstructed part of the old city of Xanthi [11]. Within the 3D scene, the user can perform queries-by-example by selecting an object. An interactive map of the 3D scene is used to inform the user about the spatial coordinates of similar to his/her query objects. Additional, the same map is used to indicate the position of the user within the 3D scene (Fig. 5).

The client side of the search engine has been built using the Quest 3D VR development platform while the server side remains technologically similar to the previous search engine. Furthermore, as the search engine uses the ASB descriptor, only objects with an axial symmetry can be indentified. Although, the case study presented in this work is domain specific, the proposed idea can be easily adopted in other domains. The search engine can be found at http://polymnia.ipet.gr/akoutsou/xanthi.

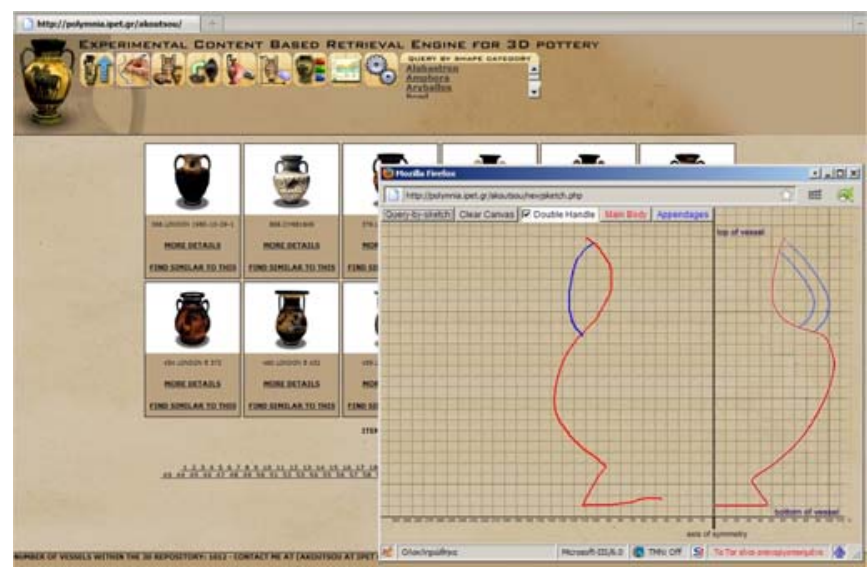

Figure 5. 3D Pottery Search Engine - Performing query-by-sketch

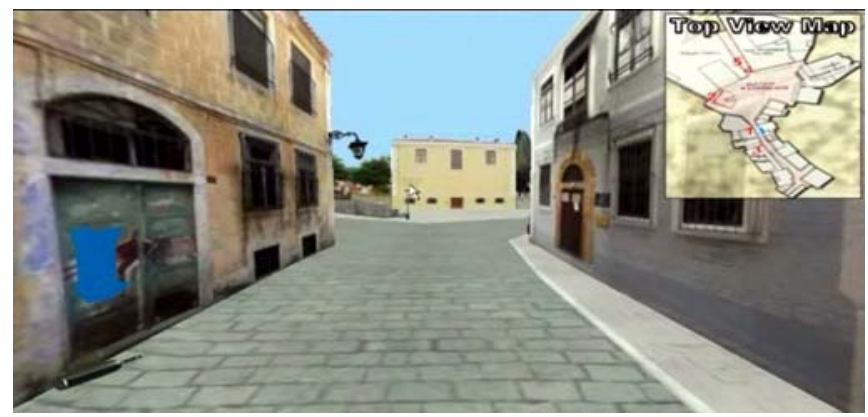

Figure 6. 3D content bases retrieval in a VR enviroment

\section{CONCLUSIONS}

In this work, we presented the idea of performing shape matching of complete or nearly complete $3 \mathrm{D}$ vessels by using content based retrieval mechanisms. We presented two descriptors that exploit the rotational symmetry found in vessels. The evaluation results indicate that the creation of domain specific descriptors is an important research field as the discriminating ability of a descriptor can be improved when the morphological features are exploited. We presented an experimental 3D pottery search engine and we attempted the integration of a content based retrieval search engine with an interactive 3D virtual reality environment. In the near future, we will attempt to evaluate the performance of descriptors that encode the depth map images using 2D shape descriptors such as 2D Fourier descriptor, 2D Zernike and Krawtchouk moments.

\section{ACKNOWLEDGMENTS}

The authors would like to thank the Cultural Heritage Unit of the Cultural and Educational Technology Institute/R.C. 'Athena' for their support in this work, Dr. Horn-yeu Shiaw, Prof. Robert Jacob and Prof. Gregory R. Crane from Tufts University, the Centre of Advanced Spatial Technologies of the Hampson Museum, Carlos Hernández Esteban and Francis Schmitt from Télécom Paris for providing us with copies of their digitised 3D vessel collections.

\section{REFERENCES}

[1] D. Gorisse, M. Cord, M. Jordan,S. Philippfoliguet, F. Precioso, "3D Content-based retrieval in Artwork Databases", in procs. 3DTVConference, May 2007.

[2] H. Shiaw, J. K. Jacob, R.G. Crane, "The 3D vase museum: a new approach to context in a digital", in procs. International Conference on Digital Libraries, June 2004.

[3] C. H. Esteban, F. Schmitt, "Image-based 3D Models Archive", http://www.tsi.enst.fr/3dmodels, Télécom Paris, 2004.

[4] A. Payne, K. Cole, K. Simon, C. Goodmaster, F. Limp, "Designing the next generation virtual museum: Making 3D artifacts available for viewing and download", in procs. of CAA 2009 Making History Interactive, March, 2009.

[5] A. Koutsoudis, G. Pavlidis, V. Liami, D. Tsiafakis, C. Chamzas, "3D pottery content-based retrieval based on pose normalisation and segmentation, Journal of Cultural Heritage, in press.

[6] A. Koutsoudis, G. Pavlidis, F. Arnaoutoglou, D. Tsiafakis, C. Chamzas, "qp: A Tool for Generating 3D Models of Ancient Greek Pottery", Journal of Cultural Heritage, Elsevier, vol. 10, issue 2, 2009.

[7] M. Chaouch, A. Verroust-Blondet, "A New Descriptor for 2D Depth Image Indexing and 3D Model Retrieval", in Procs. of ICIP 2007 September 16-19, San Antonio, Texas, USA.

[8] S. Needleman, C. Wunsch, "A General Method Applicable to the Search for Similiraties in the Amino Acid Sequence of two proteins", Journal of Molecular Biology, vol. 48, no. 3, 1970, pp. 443-453.

[9] 3D Shape Retrieval Contest, http://www.aimatshape.net/event/SHREC

[10] D.-Y. Chen, X.-P. Tian, Y.-T. Shen, and M. Ouhyoung, "On visual similarity based 3D model retrieval", Computer Graphics Forum, Vol. 22(3), September 2003, pp. 223-232.

[11] A. Koutsoudis, F. Arnaoutoglou,C. Chamzas, "On 3D Reconstruction of the old city of Xanthi", Journal of Cultural Heritage, Volume 8, Issue 1, January-March 2007, pp. 26-31. 